\title{
Study on the appearance design of horizontal polyurethane sponge cutting machine
}

\author{
Wenming Liu ${ }^{1}, \mathrm{Hao} \mathrm{Cui}^{{ }^{*}}$ \\ Shenyang Jianzhu University, Shenyang, Liaoning, 110168, China
}

\begin{abstract}
Through understanding and comprehension of working principle and structure layout of polyurethane sponge cutting machine, we conduct design and remolding on modeling and appearance of current product. Based on methods such as aesthetic principles of artistic modeling, modular design methods, modeling design methods of mechanical product, remolding and design of overall appearance of machines are studied to realize more modern aesthetics and visual impact of new appearance and promotion of the product's outward appearance quality.
\end{abstract}

\section{The working principle of polyurethane sponge cutting machine and structure layout}

Starting from the structure of upright and crossbeam, we can divide the existed machines into three kinds, namely, opening type, " $\Gamma$ " type, gantry type. As the gantry type is more common, this design of the gantry sponge cutting machine focuses on the appearance of the transformation research. From the angle of blade layout, it can be divided into single blade reciprocating cutting, double blade reciprocating cutting and ring blade cutting. The blade structure exerts prominent effects on the working efficiency of cutting machine, which also affects the space structure of whole product because of the difference in cutting size and cutting variation.

The horizontal cutting of annular blades is always used more in gantry cutting machine, which is essential for adding a certain of subsidiary structures to finish a serious of cutting work. The subsidiary structures normally include the engine case, grinding, roller, control panel and sponge collection device, all of which need to be coordinated well to finish the sponge cutting work efficiently, followed by determining the main structure of cutting machine. This design does not affect the machine cutting work on the basis of the gantry type polyurethane sponge cutting machine appearance transformation.

\section{Exterior analysis upon polyurethane sponge cutter}

The hull of polyurethane sponge cutter is capable of protecting the internal structure firstly, which is also a kind of safety protection for the staffs to prevent accidents. On the other hand, operators would generate various feeling directly, pushing the psychological hint, such as security sense, comfort sense etc.

In this section, we conducted the analysis of existing gantry type sponge cutter. The main problem with the exterior lies in the poor sealing of the shell, and part of uncovered structure lacks of stability. Because the shell area is divided into more parts, increasing the use of bolts and disassembly difficulty, and the appearance is relatively simple, thus lacking beauty to certain degree. It is required in the research process to facilitate the maintenance, replacement and disassembly of the shell.

\section{The modification research of polyurethane sponge cutter exterior}

After analyzing all the disadvantages of sponge cutter, we first can distinguish the different functions into relevant modules according to the design of modularization. Next, we need to take the humanize modification according to the disadvantages and function shortage, and then make more innovations of different modules according to the principle of beauty in form. In the whole designing process, it needs to pay attention to obeying ergonomics, so as to make the whole exterior design not only enjoy more progress on sight but also more rational on structure.

\subsection{Exterior modification research based on modularization design}

So-called modular design means to make rational design by taking modules as the functional unit and manufacturing basis, according to the functional advantages and disadvantages of existing products [1]. The modules mean to form a more complex system by combing with certain rules. The system expresses the function request of product, dividing the system into different parts which consist of modules. All the 
independent parts are treated as product's modules, which then generate the products with different modules [2].

For the complex product, it is required to separate the products into a serious of modules according to different functions, different properties and different specifications. After collecting different modules, we get various products with different structures and functions. In order to avoid the module division errors of complex products and improve the division speeds, the module pre-division of complex products should be carried out first [3]. The module pre-judge means to process the modules based on the engineer experience and principle of independence. As the specifically judged modules, we need to reduce its imported quantity and complexity level so as to make them brief, summarized and representative. According to the different functions of each part of the cutting machine, it can be divided into three parts, namely, column and beam module, tool rest module and workbench.

Analysis of upright crossbeam shows that the upright crossbeam structure determines the whole exterior of cutter, standing the torque force, bending force, so it requires its rigidity more. Then we need to pay more attention on how to choose the suitable material and how to connect column beam volume and column beam to ensure modules' stability.

There are structures on the upright, such as slide (so that the blade adapter can slide upward and bend), screw, decelerator, etc., and protective cover is added on the upright to keep operator be secure, improving its beauty at the same time.

Crossbeam has operating buttons, lighting, product logo etc. In the process of controlling the machine, operators cannot press the buttons easily, which is required to re-locate the layout during design the machine. According to man-machine engineering, the height and position of the layout are in line with the operation size of human body, and the button operation is more simple and convenient.

\subsubsection{Highlight points of upright modification. On the} basis of the function and structure of the column, the cuboid of the foundation shape was evolved to enhance the sense of the structure of the column. The exterior design of upright needs to focus on the details, including the process of cube surface, design of column edge, connected point design with crossbeam, connected point design with foundation.[4]

1. A study on the proportion of column form. The function determines structure. The upright plays a role in sustaining cutter structure. This determines that the vertical dimension of the column is much larger than the horizontal dimension. On the upwards direction, upright is the main sight, whose exterior and ratio design exert much effect on whole beauty, and the functional and aesthetic requirements should be taken into account in the selection of proportion.

2. Strengthen the whole shape change and the sense of rhyming. First, the method of "concave and convex" is adopted. The front and rear surfaces of the column are processed into regular concave and convex surfaces. The visual light and shadow relationship are used to enhance the sense of rhythm. The second one is the method to change the part of upright, such as changing one of the both side, such as size, decorative pattern or process.

3. We can take the smooth corner to finish turning direction in turning point, to avoid the potential risk caused by sharp things. As to the connected point of upright, crossbeam and bottom, we can learn from how ancient Chinese deal with the window turning point, collect the elements of symbol making the parts more outstanding. Additionally, we can pay more attention to the detailed design and make the exterior more abundant. For the bottom, we can learn from the characteristics of Roman Columns and other traditional pillars to reflect certain stability and aesthetics.

\subsubsection{Cross - beam appearance transformation}

focus. After understanding the functional characteristics of the beams, the design was carried out on the front facade. Richness is considered at the same time, along with reasonable transformation of the operation buttons, lights, factory standards and so on. As for the operating buttons, it can design the button independently. Concerning about the function, it is beneficial for operators to process as the concentrated buttons. Moreover, the height and locations make it easier for operators to operate. As shown in Figure 1, For the angle of exterior, the outstanding part means the pursuit of harmonious and unified changes, rich overall shape. At the same time, it can be endowed with different colors from the main body to enhance the visual impact [5].

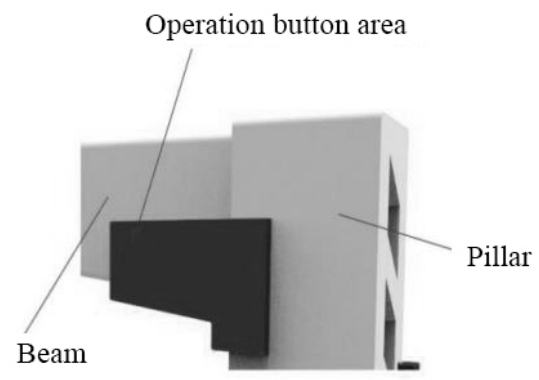

Figure 1. Schematic diagram of button area structure

\subsection{Exterior design and contrast of external appearance according to Form Beauty Law.}

According to the principle of artistic modeling aesthetics, if we modify the appearance of cutter, that is, combining the relevant theory with the actual product appearance, the exterior design will be finished reasonably. Commonly, 3 kinds of expressing skills are included in exterior appearance design, namely, size and ratio, balance and stability, unity and change. All of them play a basic role in making the product exterior design more beautiful[6].

3.2.1. Application of scale and proportion. In the process of exterior design, the issue of good proportional 
control and the determination of correct scale need to be considered in the designing product function so as to conduct further rationalization analysis accordingly, rather than inferring the proportion or accuracy independently, to avoid the mistake of cutting off the close relationship among functions. It is a way to make the exterior appearance innovative and more perfect, through considering and controlling the relation between accuracy and proportion.

Modifying the cutter's exterior appearance according to the function request, technology condition, aesthetic requirements, selecting modules of various sizes to modify the exterior, as shown in the contrasted pictures of figure 2 and figure 3.

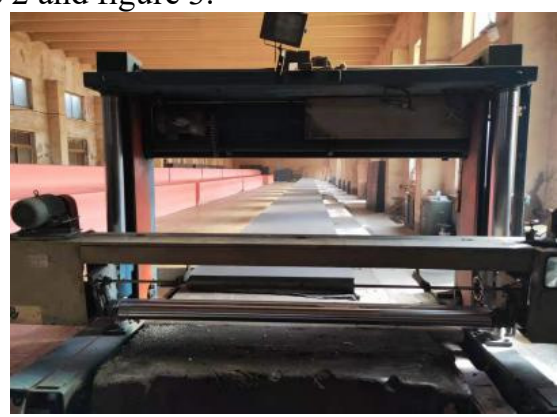

Figure 2. Cutting machine appearance chart

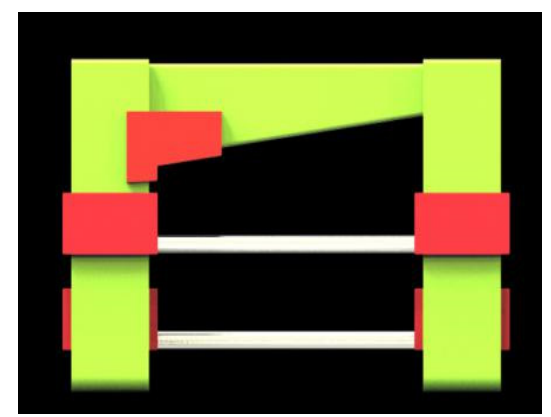

Figure 3. Design after cutting machine appearance diagram

\section{Contrast}

A set of blades have been added to the design process to increase the cutter efficiency. In the process of appearance research, there are composition considerations, ergonomics and color collocation considerations which will not describe more about added blades.

In terms of composition, to meet the practical requirements, the original cutting machine simply stacked modules of parts. The composition of pictures is mainly based on the rectangle, and the Form Beauty Law should be taken as the basis in the process of exterior modification. It takes proportion of golden rectangles with strong sense of proportion, coordination, and art, thus showing high beauty value. Compared with the previous cutter's appearance proportion, the new one can make people more comfortable in visual sensation.

In the process of exterior modification, color matching takes a large proportion. A variety of color collocation has different external results and psychological hints. The previous appearance was blue and gray. Blue is a cool tone, which makes the machine more oppressive and bring a cold sense of robot by combing with gray. The modified machine color would be yellow, a kind of bright color which can be recognized by people, symbolizing peace, glory, dignity, hope etc. For example, it is always used in traffic lights, warning boards and dangerous signals. To reduce the cold sense of machine and strengthen the warning, yellow has been chosen. As cutters have some potential risk, the red lump has been added onto the machine to remind people of keeping the intense sense.

3.2.2. The effect of balance and stability. The study on volume equilibrium is essential stage in exterior design. We can start with the evaluation of the volume balance among modules, and used the Lever Balance Principle to judge if it was balance, that is, judging if the whole balance was okay by conducting researches about if the torques at both ends of fulcrum on the bearing surface are equal. The common balance can be divided into several kinds, such as, the balance of equal quantity and different shape (Top left). The balance of quantity and shape is the same (Top right), with the balance of different quantities and shapes (bottom left), balance of different shapes and the same quantities (bottom right) etc. If the volume sensation of a black cube is 4 times that of a white cube ( 1 black $=4$ white), then figure 4 will be used to represent various forms of volume balance.

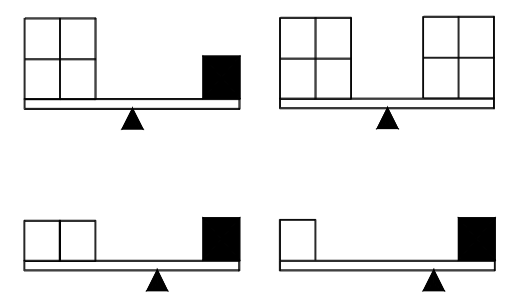

Figure 4. Schematic diagram of the equilibrium state

As shown in Figure 4, from the point of support, the balance of equal quantity and different shapes (Top left), and the balance of quantity and shape are the same (Top right). The fulcrum of these two equilibrium states is at the midpoint. All are in line with the general habit of observing objects, although you can get equilibrium in the other two states. From people's observation habits, it gives people an intuitive feeling which is more imbalance and insecurity, helping explain why the first two balanced forms are often used in the process of product appearance modeling composition.

Contrast

We should not only achieve the formal balance, but also consider it from the perspective of balance. Physical balance should be maintained to bring users a sense of balance, stability, security and other psychological feelings. In the design process, the pillars on both sides of the cutting machine are symmetrical with the same color. On the whole, the overall balance of the cutting machine is stable.

The balance of the existing product is equal to the same size and shape. This method is used in the process 
of reconstruction. This form of balance is easier to achieve and visually easy to accept.

3.2.3. Forms of harmonization and change. In the product design process, achieving a variety of artistic effects in the composition also brings the overall coordination and unity. Unity can enhance the product organization, bringing harmonious aesthetic feeling. However, if there is only unity in the shape without change, it will make people feel dull and boring. It is necessary to add certain regular or irregular changes in the harmonious and unified appearance modeling to bring about the visual impact among viewers, to form a sense of beauty, and so as to enhance the activity of the product image.

\section{Contrast}

As shown in figure 5, the exterior design adopts the technique of echo unity. Compared with the original cutting machine appearance, the whole is more harmonious and unified with more rhythm. In the process of shaping the appearance of polyurethane sponge cutting machine, the same or similar processing techniques are adopted by different components, which makes the linear, color, size, material and function of different modules have a certain form of unity. By using the same elements repeatedly, the artistic Mutual echo effect of each part is achieved. At the same time, the red color block is added to the large color block based on harmony and unity, giving people different visual feelings and make them more moved. This formed two completely different artistic styles and expressive effects from the accumulation of the original cold-toned color blocks.

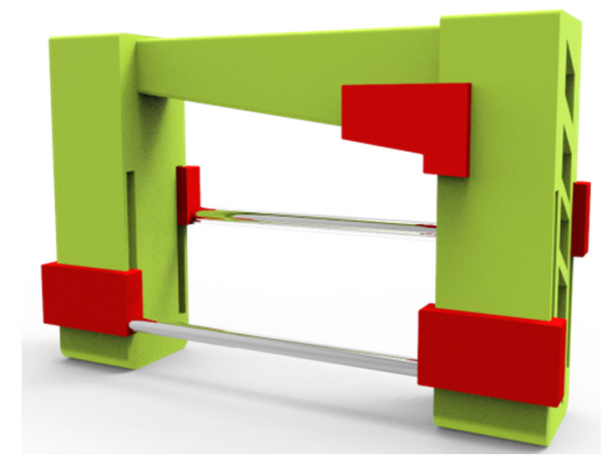

Figure5. Appearance modification rough model

\section{Conclusion}

Through remolding outward appearance of polyurethane sponge cutting machine and researches on its working principle and structure layout, advantages and disadvantages of current product are analyzed and summarized. Original advantages are retained and disadvantages are remolded on the basis of methods of aesthetic principles of artistic modeling, modular design methods and modeling design methods of mechanical product to acquire better coordination and unity between external form and its functional structure, which provides a more pleasant appearance modeling. After remolding, not only the aesthetics of the product's appearance and modeling, but also its operation safety and maintenance convenience get to be improved, which has given considerations to the spiritual and emotional demands of users when operating the machine.

\section{Reference}

1. Siyi Li (2006). Modern design methods [M], xidian university press.

2. Li-tao Pang (2016). Research and development of special container digital design system based on Creo [J]. Journal of tianjin university .

3. Junpeng Li(2012). Research on complex product module planning method for mass customization [J]. Journal of hefei university of technology .

4. Liren Wang(2012). Modeling and structural design of horizontal foam cutting machine [J]. Journal of shenyang jianzhu university .

5. Fie Liu(2015). Research on appearance design of small electric vehicle $[\mathrm{J}]$. Journal of $\mathrm{xi}$ 'an university of technology.

6. Shaohua Wan(2008). Structure and process of electronic products [M]. Beijing: Beijing university of posts and telecommunications press. 